\title{
New methods in mammary gland development and cancer: proteomics, epigenetics, symmetric division and metastasis
}

\author{
Mohamed Bentires-Alj', Marina Glukhova², Nancy Hynes' and Maria dM Vivanco*3
}

\begin{abstract}
The European Network for Breast Development and Cancer (ENBDC) meeting on 'Methods in Mammary Gland Development and Cancer' has become an annual international rendezvous for scientists with interests in the normal and neoplastic breast. The fourth meeting in this series, held in April in Weggis, Switzerland, focused on proteomics, epigenetics, symmetric division, and metastasis.
\end{abstract}

\section{Introduction}

Speaking of Weggis, where the European Network for Breast Development and Cancer (ENBDC) meeting is held, Mark Twain said 'Sunday in heaven is noisy compared to this quietness'. Indeed, people working on breast development and cancer took full advantage of this wonderfully quiet and beautiful place again this year for in-depth discussions. The meeting, held in April, started with an inspiring keynote lecture by Nancy Hynes and included the following sessions.

\section{Proteomics session}

\section{Chair: Marina Glukhova}

The proteomics session consisted of two lectures, one by Bernd Bodenmiller (University of Zurich, Switzerland) and the other by Arzu Umar (Erasmus University Medical Center, Rotterdam, The Netherlands). Bodenmiller talked about mass cytometry, a new technology that combines flow cytometry and atomic mass spectrometry and that allows simultaneous measurement of up to 100 parameters [1]. This method allows the analysis of complex regulatory networks activated in response to various

*Correspondence: mdmvivanco@cicbiogune.es

${ }^{3} \mathrm{CIC}$ bioGUNE, Cell Biology \& Stem Cells Unit, Technological Park of Bizkaia, 801 A, 48160 Derio (Bizkaia), Spain

Full list of author information is available at the end of the article environmental signals at the single-cell level and can provide a system-wide picture of signaling events. Cellular signaling intermediates are recognized by antibodies, as used for other techniques, but become labeled with rare earth metals, non-radioactive and non-biological, instead of fluorochromes, and this generates a significantly larger range of reporter tags. In the mass cytometer, cells are introduced into the plasma, atomized, and analyzed by mass spectrometry. At present, a shortage of suitable antibodies against cellular signaling compounds is one of the limiting factors in mass cytometry. Nevertheless, the method significantly extends the capacities of currently used techniques and will play a central role in the analysis of complex cellular interactive networks [2].

Umar presented a paper on the identification of a prognostic protein profile for triple-negative breast cancer. This group compared 25 triple-negative tumors that have a poor clinical prognosis with 38 tumors that have a more positive prognosis in order to define a protein signature for tumors with a high probability of relapse. The tissue samples were obtained by laser capture microdissection, and comparative proteomic profiling revealed 66 differentially expressed proteins, which led to the establishment of a 15-protein signature with predictive value [3]. The data were validated by using tissue microarrays. Umar emphasized that careful control of tissue sample preparation (digestion, reduction, and alkylation) is required in proteomic analysis in order to obtain reproducible and reliable results.

\section{Epigenetics and breast cancer \\ Chair: Nancy Hynes}

Epigenetic alterations leading to changes in gene regulation play a critical role in cancer development. These alterations include changes in the chromatin landscape contributing to modifications in DNA methylation and chromatin as well as changes in transcription of mRNA, non-coding RNAs, or microRNAs (miRNAs). Approximately $70 \%$ of patients with breast cancer present estrogen receptor (ER)-positive tumors, the vast majority of which require ER activity for their proliferation. 
Tamoxifen therapy has been the mainstay in the treatment of ER-positive tumors. Unfortunately, endocrine therapy resistance often arises, and a current high priority in the clinic is to understand why this occurs and on what basis patients should be selected for alternative therapy. Antoni Hurtado (Centre for Molecular Medicine Norway, Oslo, Norway) spoke about the important role of the transcription factor FOXA1 in mediating ER function. FOXA1 is part of the gene signature that distinguishes ER-positive from -negative breast tumors [4]. Hurtado presented ChIP-seq (chromatin immunoprecipitation followed by sequencing) data that show that FOXA1 is required for most ER-binding sites on the chromatin and that clearly show the importance of this pioneer factor for ER-responsive gene expression. Moreover, he discussed data on the sites recognized by the tamoxifen-bound ER, which overlap with the majority of estradiol-ER sites [5]. These exciting results may help explain why high FOXA1 levels are correlated with a good response to tamoxifen. In the future, it will be important to see whether FOXA1 sites are also occupied when cells are exposed to aromatase inhibitors and whether FOXA1 has a general role in endocrine therapy resistance.

In a second presentation, Heidi Dvinge (CRUKCambridge Research Institute, UK) talked about the biases inherent in different miRNA quantification platforms and the problems that can arise in the analysis of miRNA data from studies involving high-throughput sequencing and microarray expression platforms. Different miRNAs are deregulated in different tumors; by properly analyzing them, it should be possible to define networks of miRNA-regulated genes that can be examined for their possible roles in cancer. Dvinge discussed the factors that can contribute to changes in cellular miRNA content and noted that many clinical and histopathological variables have limited systematic impact on miRNA expression. By comparison, lymphocytic infiltration of primary tumors and different signaling pathways appear to be more significant determinants of miRNA levels. Finally, she discussed the Molecular Taxonomy of Breast Cancer International Consortium (METABRIC), an international consortium that is integrating genomic and transcriptional breast cancer data to further classify breast tumors into subgroups that should help determine optimal treatment of individual patients [6].

\section{Symmetric division}

\section{Chair: Maria dM Vivanco}

Stem cells are characterized by capacities to self-renew and to differentiate, and the mechanisms that control switching of symmetric and asymmetric division are of great interest in stem cell biology. Ben Simons (University of Cambridge, UK) and Salvatore Pece (IFOM-IEO
Campus, Milan, Italy) shed some light on this issue. Simons, a physicist by training, provided an excellent example of the power of interdisciplinary research by using statistical physics to address the regulation of the delicate balance between proliferation and differentiation in stem cells. He talked about general strategies of stem cell renewal which could maintain population asymmetry either in a cell-autonomous manner or under the influence of the local microenvironment. Simons discussed evidence from lineage-tracing studies of stochastic stem cell fate in adult tissues in a variety of tissue types. For example, interfollicular epidermis represents a simple model in which a single progenitor cell population maintains tissue homeostasis following balanced stochastic fate [7]. In addition, Simons presented robust lineagetracing results in the intestinal crypt and indicated that stochastic stem cell loss is compensated by division of neighboring cells and thus progresses to monoclonality [8]. It would be interesting to ascertain relevant activities in the mammary gland as a tissue subjected to a wide range of hormonal changes and where paracrine interactions may affect stem cell fate.

Breast cancer is a very complex disease, encompassing both inter- and intra-tumor heterogeneity. Pece pondered this complexity and the cellular origin of breast cancer subtypes. He discussed results of studies that used PKH26 (a lipophilic fluorescent dye that labels relatively quiescent cells within a proliferating population), combined with the mammosphere culture method, to purify human normal mammary stem cells (hNMSCs) and to identify an hNMSC signature by global transcriptome profiling [9]. The hNMSC signature distinguished welldifferentiated breast cancers from poorly differentiated ones, which are enriched in cancer stem cells. Pece argued that, mechanistically, the intrinsically high content of cancer stem cells frequently observed in poorly differentiated breast cancers is most likely a consequence of a skewing of the physiological asymmetric mode of division of stem cells toward a symmetric one, which is ultimately the basis of the unlimited self-renewal phenotype displayed by high-grade, biologically more aggressive tumors. One paradigmatic example of such molecular circuitries that control normal stem cell homeostasis and that are misregulated in cancer is the p53 loss, which is associated with acquisition of symmetric and unlimited self-renewal ability in normal and tumor mammary stem cells [10].

\section{Metastasis}

Chair: Mohamed Bentires-Alj

Keltouma Driouch (Institut Curie, Paris, France) first discussed the propensity of particular cancer cells to colonize only specific organs. Breast cancer cells metastasize preferentially to bone, lungs, liver, and brain. The 
Driouch lab uses microarray analysis of primary tumors and metastases (a) to understand the determinants of organ tropism during metastasis, which they then validate by using reverse genetics and mouse models, and (b) to derive prognostic gene signatures, which they validate by using publicly available datasets of primary breast tumors for which the outcome for the patients is known [11]. For example, the investigators analyzed gene expression profiles of lung versus non-lung metastases and found a lung-metastasis gene signature of prognostic value. Among the genes overexpressed in lung metastases is the focal adhesion protein kindlin 1 (KIND1/FERMT1), which transduces signal from integrins. KIND1 expression in breast and other cancers correlates with lung metastases. Moreover, overexpression of KIND1 enhances breast tumor growth and migration, whereas its inhibition reduces migration and tumor initiation and prevents metastasis formation [12].

Dan Medina (Baylor College of Medicine, Houston, TX, USA) described the human in mouse intraductal (HIND) method, an in vivo model for the study of the evolution of human ductal carcinoma in situ (DCIS). Despite the heterogeneity and complexity of breast cancer, a histological continuity ranging from hyperplasia to atypical hyperplasia, DCIS, and invasive breast cancer (IBC) is common in breast tumors with IBC [13]. Within the same tumor, DCIS lesions are heterogeneous in terms of grade and expression of ER, progesterone receptor, and ErbB2, and some of these lesions will progress to IBC. Unfortunately, stable DCIS cell lines that can be manipulated ex vivo and in vivo are rare. To mimic DCIS, the Medina lab injected two human breast cancer cell lines intraductally; both DCIS.com and Sum225 cells formed DCIS-like lesions but only the former progressed to IBC [14]. They then intraductally injected human DCIS cells (obtained from biopsies) with the aim of generating additional immortalized DCIS lines that represent the heterogeneity of human DCIS. In collaboration with the lab of D Craig Allred (Washington University School of Medicine, St. Louis, MO, USA), they compared matched biopsies of human DCIS versus IBC and identified differentially expressed genes (for example, cystatin A, transmembrane protein 45A, FAT tumor suppressor homologue, stratifin, osteopontin, decorin, and MMP11). They then used the HIND method to test the role of candidate genes in tumor progression [15]. Finally, Medina described the usefulness of the HIND assay for testing the effects of targeted therapy on progression from DCIS to IBC.

\section{Conclusions}

Once again, the ENBDC annual meeting provided an excellent forum for discussion of mammary gland and breast cancer research. This year, the latest advances in proteomics technology, such as mass cytometry, were discussed along with the application of proteomics to the identification of a profile that identifies tumors with high probability of relapse. The important clinical problem of recurrent cancer, as well as the technical challenges to quantify changes in miRNAs, was also part of the session on epigenetics. The consequences of the crucial switch between symmetric and asymmetric division were discussed for both normal and neoplastic tissues; understanding the mechanisms controlling this switch may be relevant for future anti-cancer therapies. Finally, the ultimate problem of metastasis was explored by using microarray analysis and intra-mammary ductal transplantation examined as a useful tool to study tumor progression. The organization of the next ENBDC meeting in Weggis, on April 25 to 27, 2013, has already begun.

\section{Abbreviations}

DCIS, ductal carcinoma in situ; ENBDC, European Network for Breast Development and Cancer; ER, estrogen receptor; HIND, human in mouse intraductal; hNMSC, human normal mammary stem cell; IBC, invasive breast cancer; miRNA, microRNA.

\section{Competing interests}

The authors declare that they have no competing interests.

\section{Acknowledgments}

Research in the lab of MB-A is supported by the Novartis Research Foundation and the European Research Council (ERC starting grant 243211-PTPsBDC). NH is supported by the Novartis Research Foundation. MV is supported by grants from the Institute of Health Carlos III (FIS) and the Department of Health of the Government of the Autonomous Community of the Basque Country.

\section{Author details}

${ }^{1}$ Friedrich Miescher Institute for Biomedical Research (FMI), Maulbeerstr. 66, CH-4058 Basel, Switzerland. 'Institut Curie, Centre de Recherche, Centre National de la Recherche Scientifique UMR144, 26 rue d'UIm, 75248 Paris cedex 05, France. ${ }^{3} \mathrm{CIC}$ bioGUNE, Technological Park of Bizkaia, 801 A, 48160 Derio (Bizkaia), Spain.

Published: 19 July 2012

\section{References}

1. Fienberg HG, Simonds EF, Fantl WJ, Nolan GP, Bodenmiller B: A platinumbased covalent viability reagent for single-cell mass cytometry. Cytometry A 2012, 81:467-475.

2. Bendall SC, Nolan GP, Roederer M, Chattopadhyay PK: A deep profiler's guide to cytometry. Trends Immuno/ 2012, 33:323-332.

3. Braakman RB, Luider TM, Martens JW, Foekens JA, Umar A: Laser capture microdissection applications in breast cancer proteomics. Methods Mol Biol 2011, 755:143-154.

4. Perou CM, Sørlie T, Eisen MB, van de Rijn M, Jeffrey SS, Rees CA, Pollack JR, Ross DT, Johnsen H, Akslen LA, Fluge O, Pergamenschikov A, Williams C, Zhu SX, Lønning PE, Børresen-Dale AL, Brown PO, Botstein D: Molecular portraits of human breast tumours. Nature 2000, 406:747-752

5. Hurtado A, Holmes KA, Ross-Innes CS, Schmidt D, Carroll JS: FOXA1 is a key determinant of estrogen receptor function and endocrine response. Nat Genet 2011, 43:27-33.

6. Curtis C, Shah SP, Chin SF, Turashvili G, Rueda OM, Dunning MJ, Speed D, Lynch AG, Samarajiwa S, Yuan Y, Gräf S, Ha G, Haffari G, Bashashati A, Russell R, McKinney S; METABRIC Group, Langerød A, Green A, Provenzano E, Wishart G, Pinder S, Watson P, Markowetz F, Murphy L, Ellis I, Purushotham A, BørresenDale AL, Brenton JD, Tavaré S, Caldas C, Aparicio S: The genomic and transcriptomic architecture of 2,000 breast tumours reveals novel subgroups. Nature 2012, 486:346-352.

7. Clayton E, Doupe DP, Klein AM, Winton DJ, Simons BD, Jones PH: A single 
type of progenitor cell maintains normal epidermis. Nature 2007, 446:185-189.

8. Snippert HJ, van der Flier LG, Sato T, van Es JH, van den Born M, KroonVeenboer C, Barker N, Klein AM, van Rheenen J, Simons BD, Clevers H: Intestinal crypt homeostasis results from neutral competition between symmetrically dividing Lgr5 stem cells. Cell 2010, 143:134-144.

9. Pece S, Tosoni D, Confalonieri S, Mazzarol G, Vecchi M, Ronzoni S, Bernard L, Viale G, Pelicci PG, Di Fiore PP: Biological and molecular heterogeneity of breast cancers correlates with their cancer stem cell content. Cell 2010, 140:62-73.

10. Cicalese A, Bonizzi G, Pasi CE, Faretta M, Ronzoni S, Giulini B, Brisken C, Minucci S, Di Fiore PP, Pelicci PG: The tumor suppressor p53 regulates polarity of self-renewing divisions in mammary stem cells. Cell 2009, 138:1083-1095.

11. Nola S, Sin S, Bonin F, Lidereau R, Driouch K: A methodological approach to unravel organ-specific breast cancer metastasis. J Mammary Gland Biol Neoplasia 2012 May 25. [Epub ahead of print].

12. Sin S, Bonin F, Petit V, Meseure D, Lallemand F, Bièche I, Bellahcène A, Castronovo V, de Wever O, Gespach C, Lidereau R, Driouch K: Role of the focal adhesion protein kindlin-1 in breast cancer growth and lung metastasis. J Natl Cancer Inst 2011, 103:1323-1337.

13. Wellings SR, Jensen HM, Marcum RG: An atlas of subgross pathology of the human breast with special reference to possible precancerous lesions. J Natl Cancer Inst 1975, 55:231-273.

14. Behbod F, Kittrell FS, LaMarca H, Edwards D, Kerbawy S, Heestand JC, Young E, Mukhopadhyay P, Yeh HW, Allred DC, Hu M, Polyak K, Rosen JM, Medina D: An intraductal human-in-mouse transplantation model mimics the subtypes of ductal carcinoma in situ. Breast Cancer Res 2009, 11:R66.

15. Valdez KE, Fan F, Smith W, Allred DC, Medina D, Behbod F: Human primary ductal carcinoma in situ (DCIS) subtype-specific pathology is preserved in a mouse intraductal (MIND) xenograft model. J Pathol 2011, 225:565-573.

\section{doi:10.1186/bcr3216}

Cite this article as: Bentires-Alj M, et al: New methods in mammary gland development and cancer: proteomics, epigenetics, symmetric division and metastasis. Breast Cancer Research 2012, 14:314. 